\title{
Is Oral Health All About Tooth Brushing? A Longitudinal Study in Individuals With Brain Injury in A Neurorehabilitation Setting
}

Simple Kothari

Aarhus University

Gustavo Nascimento

Aarhus University

Mille Jakobsen

Aarhus University

Jørgen Nielsen

Aarhus University

Mohit Kothari ( $\nabla$ mohkot@rm.dk)

Aarhus University

\section{Research Article}

Keywords: Acquired brain injury, hospitalization, neurorehabilitation, nursing, oral health, oral hygiene, periodontitis, stroke.

Posted Date: December 7th, 2020

DOI: https://doi.org/10.21203/rs.3.rs-112535/v1

License: (c) (1) This work is licensed under a Creative Commons Attribution 4.0 International License. Read Full License 


\section{Abstract}

Objective: To investigate oral health changes and its associated factors during hospitalization in individuals with acquired brain injury $(A B I)$.

Material and Methods: Sixty-one individuals were recruited to evaluate the acute changes in oral health by performing plaque, calculus, bleeding on probing (BOP) and bedside oral examination (BOE) at week 1 and week 5. Individuals' brushing habits, eating difficulties, onset of pneumonia etc. were retrieved from e-journal. Association between oral-health outcomes to systemic variables were investigated through multilevel regression models.

Results: Dental plaque $(P=0.01)$ and total $B O E$ score $(P<0.05)$ decreased over time but not the calculus $(P=0.30), B O P(P=0.06)$ and increase in tooth brushing frequency $(P=0.06)$. Reduction in plaque, and BOE over time were negatively associated with higher score of periodontitis at baseline (coef. -6.8; -1.0, respectively), which in turn were associated with an increased proportion of BOP (coef. $\approx 15.0$ ). Increased proportion of calculus was associated with eating difficulties (coef. 2.3) and onset of pneumonia (coef. $6.2)$.

Conclusions: Nursing care has been fundamental in improving oral health but non-significant improvement in calculus, BOP and brushing frequency indicates a need for development in existing oral care program through academic-clinical partnership keeping eating difficulties and patients' vegetative and cognitive state in consideration.

\section{Introduction}

Oral care is essential to maintain oral health and prevent complications such as periodontal diseases and tooth loss in patients with acquired brain injury $(\mathrm{ABI})^{1-3}$. Poor oral hygiene among dependent hospitalized patients could lead to severe complications such as poor nutritional intake, increased length of hospital stay, and pneumonia ${ }^{1,4,5}$. In relation to oral health, stroke can cause hemiparesis and hemiplegia to the facial muscles and the muscles of the pharynx, tongue, palate, and mastication, resulting in impaired oral clearance ${ }^{6,7}$. Medications prescribed for patients after stroke may further impact oral health resulting in, for example, dry mouth, oral ulcers, and stomatitis ${ }^{8}$. ABI individuals with swallowing difficulties have compromised oral clearance and leads to increased bacterial load ${ }^{1}$. Swallowing impairment, along with poor oral health has a significant impact on an individual's nutritional intake ${ }^{9}$, increasing the risk of aspirational pneumonia ${ }^{5,10}$, which in turn has a negative impact on rehabilitation and other functional outcomes ${ }^{5,11}$.

Evidence suggests that stroke survivors with increased plaque and bacterial load experience a deterioration of the periodontal conditions ${ }^{12,13}$. Recently, a study showed that $40 \%$ of the $A B I$ population had an abundant amount of dental plaque and increased bleeding on probing (BOP), a finding that may indicate an acute hospitalization effect ${ }^{3}$. In addition, $74 \%$ of the $A B I$ individuals also had severe 
periodontitis, a condition, supported by their poor socio-behavioral and medical history, representing a chronic stage of an oral health disease ${ }^{3}$.

Post $A B I$, many patients are reliant on nursing staff to assist them with oral hygiene. Despite indications that healthcare staff is interested in improving this aspect of care, a recent survey among $>250$ health professionals showed that oral care had not been their prime focus due to barriers such as, lack of time due to prioritizing other emergency tasks, unfocused oral care policies, lack of training and evidencebased continuing education ${ }^{14}$.

In the light of the current evidence on the importance of oral health among individuals with $\mathrm{ABI}$, oral care management through oral care providers could play an important role in this area ${ }^{9}$. It is not clear whether the oral care provided by the healthcare professionals help improve or deteriorate the oral health in a neurorehabilitation setting $3,14,15$. This new knowledge may provide an overview to promote and manage oral health in individuals with $A B I$ in a neurorehabilitation setting. Accordingly, the aim of this study was to investigate the changes in oral health status over time (5 weeks) and its associated factors during hospitalization in patients with $A B I$. We hypothesized that the current standard oral care provision requires further structural improvement and modifications.

\section{Results}

Of the 90 patients included at baseline, 61 provided data for the 5-week follow-up. The mean age was 55.1 years $( \pm 14.0)$, and $64 \%$ of the patients were male. More information about the sociodemographic data of the participants can be found elsewhere ${ }^{3}$.

Paired analysis revealed that the proportion of sites with visible dental plaque $(P=0.01)$ significantly decreased over the time but BOP $(P=0.06)$, calculus $(P=0.30)$ and the frequency of tooth brushing $(P=$ 0.06) did not achieve statistically significant changes after 5 weeks of hospitalization. In addition, the total BOE score $(P<0.001)$ significantly improved over time and also most of the BOE domains like, swallow, saliva, mucosa, teeth and odor $(P<0.05)$ (Table 2).

Mixed-effects regression models indicated that individuals with 'moderate' periodontitis at baseline (coef. -6.8) and those hospitalized at the regional ward (coef. -15.6) had decreased proportion of sites with dental plaque. In addition, the number of extracted teeth (coef. -1.0), the proportion of calculus (-0.5), and the time (-8.8) were also associated with a reduction in the proportion of plaque (Table 3A).

Patients with higher scores of both 'moderate' (coef. 14.3) and 'severe' (coef. 15.6) periodontitis at baseline had an increase in the proportion of sites with BOP over the study period (Table 3B), whereas those who improved their 'cognitive' domain (coef. -6.6) had a decrease in the proportion of BOP. The number of extracted tooth at baseline (coef. 0.5) and increased proportion of plaque over the study period (coef. 0.4) were also associated with an increased proportion of BOP after the 5-week follow up. As displayed in Table 3C, those who developed pneumonia during hospitalization (coef. 6.2) and those with 
eating difficulties over the study period (coef. 2.3) had an increase in the proportion of sites with dental calculus.

Finally, mixed-effects regression models indicated that the individuals with higher scores of 'moderate' periodontitis at baseline (coef. -1.0), those hospitalized at the regional ward (coef. -1.7), and those who improved their 'motor' skills during the study period (coef. -0.6) had a reduction in their total BOE score, whereas those with dysphagia at baseline (coef. 0.5) and the old individuals (coef. 0.04) had an increased total BOE score after five weeks (Table 3D).

\section{Discussion}

The main finding of the study was that the oral health parameters such as visible plaque and BOE scores significantly improved during 5 week stay at neurorehabilitation setting but the proportion of sites with BOP and frequency of tooth brushing over time (5-week stay) did not reach statistical significance. These findings demonstrate that although there was an improvement in the oral health status in hospitalized individuals, it was not substantiated, indicating a need for further development in oral care program.

A significant reduction in the amount of dental plaque was observed over time. Plaque is a biofilm comprised of diverse community of microorganisms which is formed regularly on the tooth surface and can be maintained by proper tooth brushing and flossing 1,16,17. Individuals with less severe ABI are usually admitted to the regional ward at HNRC instead of highly-specialized ward due to their better motor and cognitive functions, which makes them more co-operative then severely affected individuals with $A B I^{18}$. It was also evident from the mixed regression analysis that individuals admitted to the 'regional ward', showed an strong association in reduction of plaque compare to highly-specialized ward 19. Interestingly, the proportion of plaque was also reduced in individuals with 'moderate' periodontitis and with an increased proportion of calculus and BOP, indicating that there are also other factors such as host-immune response ${ }^{5}$, which were not taken into account in this study, that might have influenced BOP.

Gingival bleeding (BOP) is an acute reversible inflammatory condition that occurs as a response to plaque accumulation on the periodontal tissues ${ }^{20}$. In general, good oral hygiene practices are sufficient to control and reduce gingival bleeding ${ }^{17,21}$, which was also shown in the current study with strong association between plaque and BOP (Table $3 \mathrm{C}$ ). However, despite the significant plaque reduction, proportion of sites with gingival inflammation (BOP) did not reduce in the same individuals over the study period. It is also important to discuss that the SD values of BOP were probably quite higher as few individuals had a very low BOP, while some (especially those with periodontitis) had high BOP. Such a finding suggests that factors other than plaque might play a role in the onset and progression of gingival inflammation. It has been shown that a more exacerbated and rapid immune response, acute hospitalization and cognitive and systematic complications are linked to a higher neutrophilic activity, which is able to mount an immediate response when exposed to plaque ${ }^{22,23}$. Interestingly, our findings demonstrated that BOP decreased over time in individuals who showed an improvement in their 'cognitive' function, indicating reduction in confusion and agitation leading to increased cooperation with 
oral care, which very well correlates with previous research ${ }^{5}$. It has been shown that BOP is closely associated with 'severe' periodontitis, which in addition to an already existing cognitive impairment, may contribute to other chronic conditions that share a common biological background to $A B I 5,24$. Assuming that such an exacerbated immune response is not restricted to the oral cavity, this may interfere with other inflammatory processes, especially in a hospital setting and in the presence of other comorbidities, explaining partially our findings ${ }^{5}$. On the other hand, the proportion of sites with BOP increased over time among patients with both 'moderate' and 'severe' periodontitis, despite the increase in tooth brushing frequency over the same period. This finding indicates that the oral health status in these patients were poor and tooth brushing alone may not be enough to tackle oral health problems. Despite the efforts made by nurses to maintain oral hygiene, there was still deterioration of the inflammatory periodontal condition ${ }^{1}$. This suggests the need for involvement of dental personnel in hospitals for providing adequate oral care to $\mathrm{ABI}$ patients ${ }^{3}$.

Calculus, defined as hard deposit around the gingiva as a result of long-term plaque accumulation, showed no significant improvement over time. It is important to highlight that calculus does not indicate disease, but it makes oral hygiene more difficult to maintain as well as works as a plaque-retaining factor 25. Even though the removal of calculus is not possible without professional dental assistance, it is possible to maintain proper oral hygiene by preventing calculus formation. Such a finding supports the idea that chronic oral changes require professional help from dental personnel as well as changes in socio-behavioral factors for the improvement of oral health ${ }^{20}$. Our findings also revealed that individuals with eating difficulty and those who developed pneumonia during hospitalization had an increase in the proportion of sites with dental calculus. One may speculate whether the combination of dental calculus and eating difficulties may influence the onset of pneumonia. A recent study on patients with $A B I$ has shown a robust association between periodontitis and debilitating conditions like dysphagia, dependency on a feeding tube, which is a major concern, as they lead to pneumonia ${ }^{5}$. Although our study does not allow us to disentangle the causal relationship between these conditions, our overall findings suggest the need for increased focus on oral care especially for $A B I$ individuals with conditions like eating difficulties and severe cognitive disturbances.

Interestingly, BOE scores decreased in individuals with higher scores of 'moderate' periodontitis. As discussed, 'moderate' periodontitis originates essentially from neglected oral hygiene, so does most the BOE domains $3,5,21,26$. Thus, the combined effect of plaque reduction and increased frequency of oral hygiene can explain this association. It should be noted that, although BOE is a simple and easy to use tool in hospital settings, especially in intensive care units, its usefulness is questioned in ABI patients and therefore, the BOE results may be carefully interpreted ${ }^{2,3}$. This is because the instrument seems not to reflect the real clinical conditions of patients with $A B I$, thus, affecting the treatment plan. Further, it has been shown that 'ageing' patients have more compromised function than young individuals, making them more vulnerable to dysphagia and unable to perform and maintain good oral hygiene procedures 3,5 . 
A recent survey conducted among 157 oral caregivers at HNRC showed that the majority of oral caregivers were aware of the existing 'Danish National Clinical Guidelines for Oral Care' ${ }^{19}$. However, a significant number of oral care providers did not follow the guidelines systematically, expressing it as an

ineffective, time-consuming, and difficult to follow ${ }^{14}$. Professionals were aware that patients with eating difficulties have challenges and different requirements ${ }^{14}$ and on top cognitive and motor deficits adds an extra challenge to maintain the oral hygiene ${ }^{5}$. In addition, there is always a professional dilemma to maintain oral hygiene standard whilst respecting patient's autonomy once they refuse for the oral hygiene care, even if it is required. Therefore, all these factors should be considered while formulating and designing oral care training and guidelines to improve oral care in a neurorehabilitation setting.

\section{Methodological Considerations}

The current study sample originates from a single hospital setting, and therefore, our findings may have limited external validity. However, it is worth mentioning that this hospital is a reference center for the treatment of $\mathrm{ABI}$ patients and receives patients from most regions of Denmark. In addition, limited sample size and $30 \%$ lost to follow-up might have reduced the analytical power, as can be noted by borderline P-values. Future studies with large samples originating from several centers are needed. Another limitation of the study was short follow-up time, given the chronicity of the most common oral diseases, i.e., dental caries and periodontitis. However, treatment of these conditions demands the involvement of dental personnel with appropriate armamentarium, which was not within the scope of the study. As our purpose was to observe the effect of hospitalization on oral health, we decided to evaluate conditions such as the proportion of dental plaque and of BOP, as those parameters can rapidly change. We also need to be aware that few patients were excluded due to extreme fatigue, agitation, motorcognitive deficits etc., leaving us with no opportunity for clinical examination, which might be a bias in representing the entire oral health status.

\section{Conclusions}

A significant reduction in dental plaque and total BOE score was observed over time. However, nonsignificant improvement in gingivitis, the proportion of calculus, and brushing frequency indicate the need for a further development of oral care program in individuals with $A B I$ keeping motor-cognitive deficits and eating difficulties in consideration. This study also enforces the need for the involvement of dentist in educating and supervising non-dental professionals at an early stage to provide a better and an integrated oral care program in $\mathrm{ABI}$ individuals in hospital settings.

\section{Methods}

\section{Participants and recruitment}


All individuals with $\mathrm{ABI}$ admitted between February-June 2019 at neurorehabilitation center were recruited in a longitudinal observational study. In total, 132 individuals with $A B I$ were screened and examined within the first week (baseline) from the admission day and later at week 5 , re-examined to assess the acute changes in oral health. Out of 132 individuals, 90 were eligible for the week 1 assessment, and 61 individuals were eligible for week 5 assessment after fulfilling all the above-mentioned eligibility criteria (For a detailed eligibility criteria, see Appendix 1).

\section{Procedures and measures \\ a. Medical records}

For a detailed description, see Appendix 1 and Kothari et al 2,3,5.

\section{b. Demographics and socio-behavioral history}

For a detailed description, see Appendix 1 and Kothari et al 2,3,5.

\section{c. Motor and cognitive deficits related to orofacial function parameters}

Motor and cognitive domains related to orofacial function were collected from the subset of the following brain injury scales at both baseline and at week 5: The Early Functional Ability (EFA) scale ${ }^{27}$, the Functional Independent Measure (FIM) ${ }^{28}$, the Functional Oral Intake Scales (FOIS) ${ }^{29}$, and the Rancho Los Amigos Scale $(R L A S)^{30}$. In our previous study, by employing a factor analysis approach on the questionnaire data and were able to identify two different factors: motor domain based on their orofacial health and entire cognitive domain (For a detailed description, see Appendix 1 and Kothari et al ${ }^{5}$ ).

\section{d. Comprehensive oral health assessment}

The comprehensive oral health assessment tool used in the current study was previously developed, tested, and was thereafter modified by our group ${ }^{2,3}$. (For a detailed description, see Appendix 1 and Kothari et al ${ }^{2,3}$ ).

\section{e. Standard oral care program at HNRC}

Based on the Danish national clinical guidelines for oral care, the health care professionals follow the standard oral care program in all the individuals admitted at HNRC ${ }^{19}$. The standard oral care program was supplemented by the additional oral care depending on the individual needs. See Table 1 for details.

\section{Data Analyses}

Data on the proportion of plaque, calculus, BOP, BOE scores, and frequency of tooth brushing were submitted to descriptive analyses. In addition, paired analyses (t-test for normally distributed variables 
and Wilcoxon signed-rand test for non-normally distributed variables) were also conducted. Using multilevel mixed-effects regression models, we were able to investigate the association between changes in oral health outcomes with both time-varying, collected at both baseline and week 5 , and non-varying (elicited at baseline only) variables. To select potential confounders, we used the "backward" stepwise procedure, in which all variables were entered in the model, and then subsequently removed. Only variables with a P-value $<0.20$ were maintained in the model and those with a P-value $<0.05$ were considered statistically significant. The data analysis was carried out using the software Stata 14.2 (StataCorp., College Station, TX, USA).

\section{Declarations}

\section{Acknowledgements}

The authors would like to thank Lena Aadal, Associate Professor in Nursing, Hanne Møller Nielsen, neurorehabilitation specialized nurse, Katje Bjerrum, neurorehabilitation specialized occupational therapist and the entire nursing staff at HNRC, who were integral in potential participant identification, clinical and scientific critical discussions. A special thanks to Helle Leegård, Head Nurse and Ole Hovgaard, Clinical Head, Department of Dentistry and Oral Health, Aarhus University for encouragement and providing dental instruments for the study. The author would also like to express his gratitude to $\mathrm{Mr}$. Olaf Westening, country manager of Hu-Freidy (www.hu-friedy.eu) for donating fourteen UNC-15 periodontal probe to perform the periodontal clinical examination and without saying to all the participants who gave their time generously.

\section{Compliance with Ethical Standards}

Conflict of interest: The authors state that they have no conflict of interest.

Funding: MK received grant from Health Research Fund of Central Denmark Region for the current study with grant number A1407. The authors' salary was supported by their respective institution.

Ethical approval: The study was part of a nursing quality development project. Hence, according to the Danish law, no ethical approval was required but permission of institutional review board for clinical projects was taken. Hence, according to Danish law, there was no requirement to have informed consent from all subjects who participated in the study. This study was performed in accordance with the 1964 Helsinki declaration and its later amendments or comparable ethical standards and all methods were carried out in accordance with relevant guidelines and regulations.

\section{References}

1. Kothari, M. et al. Oral health status in patients with acquired brain injury: a systematic review. Oral Surg Oral Med Oral Pathol Oral Radiol 123, 205-219.e207, doi:10.1016/j.oooo.2016.10.024 (2017).

2. Kothari, M., Spin-Neto, R. \& Nielsen, J. F. Comprehensive oral-health assessment of individuals with acquired brain-injury in neuro-rehabilitation setting. Brain Inj 30, 1103-1108, 
doi:10.3109/02699052.2016.1167244 (2016).

3. Kothari, S. F., Nascimento, G. G., Jakobsen, M. B., Nielsen, J. F. \& Kothari, M. Oral health: something to worry about in individuals with acquired brain injury? Brain Inj, 1-6, doi:10.1080/02699052.2020.1795720 (2020).

4. Brady, M., Furlanetto, D., Hunter, R. V., Lewis, S. \& Milne, V. Staff-led interventions for improving oral hygiene in patients following stroke. Cochrane Database Syst Rev, Cd003864, doi:10.1002/14651858.CD003864.pub2 (2006).

5. Kothari, S., Nascimento, G., Jakobsen, M., Nielsen, J. \& Kothari, M. Periodontitis and orofacial healthrelated systemic impairment in patients with brain injury: A factor analysis approach. $J$ Periodontol (under review) (2020).

6. Ajwani, S. et al. Integrated oral health care for stroke patients - a scoping review. J Clin Nurs 26, 891901, doi:10.1111/jocn.13520 (2017).

7. Mortensen Jesper, P. A. R., Nielsen Jørgen Feldbæk, Kothari Mohit. Construct and content validity of the Functional Oral Intake Scale; Analyses from a cohort of patients with acquired braininjury. Brain Injury (under review) (2020).

8. Janket, S. J. et al. Xerostomic medications and oral health: the Veterans Dental Study (part I). Gerodontology 20, 41-49, doi:10.1111/j.1741-2358.2003.00041.x (2003).

9. Bailey, R. L., Ledikwe, J. H., Smiciklas-Wright, H., Mitchell, D. C. \& Jensen, G. L. Persistent oral health problems associated with comorbidity and impaired diet quality in older adults. J Am Diet Assoc 104, 1273-1276, doi:10.1016/j.jada.2004.05.210 (2004).

10. Dickinson, H. Maintaining oral health after stroke. Nurs Stand 26, 35-39, doi:10.7748/ns2012.08.26.49.35.c9233 (2012).

11. Adams, H. P., Jr. et al. Guidelines for the early management of adults with ischemic stroke: a guideline from the American Heart Association/American Stroke Association Stroke Council, Clinical Cardiology Council, Cardiovascular Radiology and Intervention Council, and the Atherosclerotic Peripheral Vascular Disease and Quality of Care Outcomes in Research Interdisciplinary Working Groups: the American Academy of Neurology affirms the value of this guideline as an educational tool for neurologists. Stroke 38, 1655-1711, doi:10.1161/strokeaha.107.181486 (2007).

12. Pow, E. H., Leung, K. C., Wong, M. C., Li, L. S. \& McMillan, A. S. A longitudinal study of the oral health condition of elderly stroke survivors on hospital discharge into the community. Int Dent J 55, 319324, doi:10.1111/j.1875-595x.2005.tb00330.x (2005).

13. Pillai, R. S. et al. Oral Health and Brain Injury: Causal or Casual Relation? Cerebrovasc Dis Extra 8, 115, doi:10.1159/000484989 (2018).

14. Odgaard, L. \& Kothari, M. Survey of oral nursing care attitudes, knowledge and practices in a neurorehabilitation setting. J Oral Rehabil 46, 730-737, doi:10.1111/joor.12799 (2019).

15. Kwok, C., Mclntyre, A., Janzen, S., Mays, R. \& Teasell, R. Oral care post stroke: a scoping review. J Oral Rehabil 42, 65-74, doi:10.1111/joor.12229 (2015). 
16. Cabre, M. et al. Prevalence and prognostic implications of dysphagia in elderly patients with pneumonia. Age Ageing 39, 39-45, doi:10.1093/ageing/afp100 (2010).

17. Ortega, O. et al. Oral health in older patients with oropharyngeal dysphagia. Age Ageing 43, 132-137, doi:10.1093/ageing/aft164 (2014).

18. Vol. 13 Medicinsk Teknologivurdering (ed Sundhedsdokumentation Sundhedsstyrelsen) (Copenhagen, 2011).

19. Trosborg, I., Christensen, L., Konradsen, H. (Aarhus: Center for Kliniske Retningslinjer. Afdeling for Sygeplejevidenskab, 2010).

20. Dai, R. et al. A systematic review and meta-analysis of clinical, microbiological, and behavioural aspects of oral health among patients with stroke. J Dent 43, 171-180, doi:10.1016/j.jdent.2014.06.005 (2015).

21. Prendergast, V., Kleiman, C. \& King, M. The Bedside Oral Exam and the Barrow Oral Care Protocol: translating evidence-based oral care into practice. Intensive Crit Care Nurs 29, 282-290, doi:10.1016/j.iccn.2013.04.001 (2013).

22. Nascimento, G. G., Leite, F. R. M., Vestergaard, P., Scheutz, F. \& Lopez, R. Does diabetes increase the risk of periodontitis? A systematic review and meta-regression analysis of longitudinal prospective studies. Acta Diabeto/ 55, 653-667, doi:10.1007/s00592-018-1120-4 (2018).

23. Nascimento, G. G. et al. Diet-Induced Overweight and Obesity and Periodontitis Risk: An Application of the Parametric G-Formula in the 1982 Pelotas Birth Cohort. Am J Epidemio/ 185, 442-451, doi:10.1093/aje/kww187 (2017).

24. Nascimento, P. C. et al. Association Between Periodontitis and Cognitive Impairment in Adults: A Systematic Review. Front Neurol 10, 323, doi:10.3389/fneur.2019.00323 (2019).

25. Jepsen, S., Deschner, J., Braun, A., Schwarz, F. \& Eberhard, J. Calculus removal and the prevention of its formation. Periodontol 2000 55, 167-188, doi:10.1111/j.1600-0757.2010.00382.x (2011).

26. Nascimento, G. G., Baelum, V., Dahlen, G. \& Lopez, R. Methodological issues in assessing the association between periodontitis and caries among adolescents. Community Dent Oral Epidemiol 46, 303-309, doi:10.1111/cdoe.12367 (2018).

27. Hankemeier, A. \& Rollnik, J. D. The Early Functional Abilities (EFA) scale to assess neurological and neurosurgical early rehabilitation patients. BMC Neuro/ 15 , 207, doi:10.1186/s12883-015-0469-z (2015).

28. Nichol, A. D. et al. Measuring functional and quality of life outcomes following major head injury: common scales and checklists. Injury 42, 281-287, doi:10.1016/j.injury.2010.11.047 (2011).

29. Crary, M. A., Mann, G. D. \& Groher, M. E. Initial psychometric assessment of a functional oral intake scale for dysphagia in stroke patients. Arch Phys Med Rehabil 86, 1516-1520, doi:10.1016/j.apmr.2004.11.049 (2005).

30. Lin, K. \& Wroten, M. in StatPearls (StatPearls PublishingStatPearls Publishing LLC., 2020). 


\section{Tables}

Table 1

Standard oral care program at HNRC

\begin{tabular}{|c|c|c|}
\hline $\begin{array}{l}\text { Individuals at } \\
\text { HNRC }\end{array}$ & $\begin{array}{l}\text { Standard oral-care (recommended clinical } \\
\text { guidelines) }\end{array}$ & $\begin{array}{l}\text { Supplemental oral care } \\
\text { (case-dependent) }\end{array}$ \\
\hline $\begin{array}{l}\text { All } \mathrm{ABI} \\
\text { individuals }\end{array}$ & $\begin{array}{l}\text { Instruction to brush twice a day, preferably after } \\
\text { each meal. }\end{array}$ & $\begin{array}{l}\text { Chlorhexidine mouth wash } \\
(0.12 \%)\end{array}$ \\
\hline \multirow[t]{3}{*}{ (self-oral care) } & \multirow{3}{*}{$\begin{array}{l}\text { Free to have any toothbrush they bring from home } \\
\text { (small head/ big head/ electric soft bristle } \\
\text { toothbrush) with fluoride toothpaste } \\
\text { (min.1450 ppm). }\end{array}$} & Oral mucosal care. \\
\hline & & Dental floss once a day. \\
\hline & & $\begin{array}{l}\text { Lip moisturizer for dry or } \\
\text { cracked lips }\end{array}$ \\
\hline \multirow{4}{*}{$\begin{array}{l}\text { ABI individuals } \\
\text { with } \\
\text { eating or } \\
\text { cognitive } \\
\text { difficulties } \\
\text { (oral care by } \\
\text { caregivers) }\end{array}$} & $\begin{array}{l}\text { Orofacial stimulation (face, lip, gum and tongue) } \\
\text { before every meal. }\end{array}$ & $\begin{array}{l}\text { Chlorhexidine mouth wash } \\
(0.12 \%) /\end{array}$ \\
\hline & $\begin{array}{l}\text { Cleaning of mouth for food debris and secretions } \\
\text { before and after each intake of food and drinks. }\end{array}$ & \multirow{3}{*}{$\begin{array}{l}\text { Oral mucosal care. } \\
\text { Lip moisturizer for dry or } \\
\text { cracked lips }\end{array}$} \\
\hline & $\begin{array}{l}\text { Use of small head soft bristle toothbrush and } \\
\text { fluoridated non-foaming toothpaste } \\
\text { (min.1450 ppm). }\end{array}$ & \\
\hline & $\begin{array}{l}\text { Tooth brushing in circulatory motion starting } \\
\text { bucally, palatally and then to occlusal table twice } \\
\text { a day after each meal. }\end{array}$ & \\
\hline \multirow{4}{*}{$\begin{array}{l}\text { Tracheotomized } \\
\text { ABI individuals } \\
\text { (oral care by } \\
\text { caregivers) }\end{array}$} & Oral care in recline or side wise position. & $\begin{array}{l}\text { Carbonated water for } \\
\text { patients with dry mouth. }\end{array}$ \\
\hline & $\begin{array}{l}\text { Orofacial stimulation (face, lip, gum and tongue) } \\
2-3 \text { times/ days. }\end{array}$ & \multirow{2}{*}{$\begin{array}{l}\text { Mouthwash containing } \\
\text { both chlorhexidine }(0.05 \%) \\
\text { and fluoride }(0.05 \%) \text { for } \\
\text { bleeding gums. }\end{array}$} \\
\hline & $\begin{array}{l}\text { Cleaning of mouth for food debris and secretions } \\
2-3 \text { times/ days. }\end{array}$ & \\
\hline & $\begin{array}{l}\text { Individual who are unable to spit, the oral cavity is } \\
\text { cleaned by mouthwash having amyloglucosidase } \\
\text { and fluoride (Zendium) with the help of sponge or } \\
\text { swabs. }\end{array}$ & $\begin{array}{l}\text { Lip moisturizer for dry or } \\
\text { cracked lips. }\end{array}$ \\
\hline
\end{tabular}


Table 2

Changes in oral health parameters during hospitalization

\begin{tabular}{|llll|}
\hline Items & $\begin{array}{l}\text { Baseline } \\
\text { (Week 1) }\end{array}$ & Week 5/ Discharge & P-value \\
\hline 1. \% Plaque $^{2}$ & $50.6(27.1)$ & $42.2(30.4)$ & 0.01 \\
\hline 2. \% BOP ${ }^{2}$ & $41.9(42.7)$ & $30.4(37.3)$ & 0.06 \\
\hline 3. \% Calculus ${ }^{2}$ & $5.5(12.5)$ & $4.7(11.9)$ & 0.29 \\
\hline 4. BOE ${ }^{1}$ & & & \\
\hline a. Swallow & $1(1-2)$ & $1(1-1)$ & $<0.01$ \\
\hline b. Lips & $1(1-2)$ & $1(1-1)$ & 0.10 \\
\hline c. Tongue & $1(1-2)$ & $1(1-2)$ & 0.29 \\
\hline d. Saliva & $1(1-2)$ & $1(1-2)$ & 0.03 \\
\hline e. Mucosa & $1(1-2)$ & $1(1-1)$ & 0.01 \\
\hline f. Gingiva & $1(1-2)$ & $1(1-1)$ & $<0.01$ \\
\hline g. Teeth & $1(1-2)$ & $1(1-1)$ & 0.05 \\
\hline h. Odor & $1(1-2)$ & $1(1-1)$ & $<0.001$ \\
\hline Total & $11(9-13)$ & $9(8-11)$ & 0.06 \\
\hline 5. Frequency of toothbrushing ${ }^{1}$ & $1.8(0.6)$ & $2.0(0.5)$ & \\
\hline 1 Wilcoxon signed ranked test; ${ }^{2}$ Paired t-test; BOE: Bedside oral examination; BOP: Bleeding on probing \\
\hline
\end{tabular}

Table 3: Mixed effect regression model comparing oral health parameters with systemic findings

Table 3A: Mixed effect regression model comparing Plaque and systemic findings 


\begin{tabular}{|c|c|c|c|}
\hline & \% Plaque & & \\
\hline Variables & Coefficient $(\beta)^{1}$ & $95 \% \mathrm{Cl}$ & P-value \\
\hline 'Moderate' periodontitis at baseline & -6.8 & $-12.1 ;-1.5$ & 0.012 \\
\hline Regional ward (Reference: high-specialized ward) & -15.6 & $-27.3 ;-3.9$ & 0.009 \\
\hline \# Extracted teeth at baseline & -1.0 & $-1.9 ;-0.1$ & 0.05 \\
\hline$\%$ Calculus & -0.5 & $-0.9 ; 0.0$ & 0.032 \\
\hline Time & -8.8 & $-15.4 ;-2.2$ & 0.009 \\
\hline
\end{tabular}

Table 3B: Mixed effect regression model comparing BOP with systemic findings

\begin{tabular}{|llll|}
\hline & $\%$ BOP & & \\
\hline Variables & Coefficient $(\boldsymbol{\beta})^{1}$ & $95 \% \mathrm{Cl}$ & P-value \\
\hline 'Moderate' periodontitis at baseline & 14.3 & $9.6 ; 19.0$ & $<0.001$ \\
\hline 'Severe' periodontitis at baseline & 15.6 & $11.6 ; 19.5$ & $<0.001$ \\
\hline \# Extracted teeth at baseline & 0.5 & $0.1 ; 1.1$ & 0.045 \\
\hline 'Cognitive' domain over the study period & -6.6 & $-11.6 ;-1.6$ & 0.010 \\
\hline \% Plaque & 0.4 & $0.2 ; 0.7$ & 0.001 \\
\hline
\end{tabular}

Table 3C: Mixed effect regression model comparing calculus with systemic findings

\begin{tabular}{|llll|}
\hline & \% Calculus & & \\
\hline Variables & Coefficient $(\boldsymbol{\beta})^{\mathbf{1}}$ & $\mathbf{9 5 \%} \mathbf{C l}$ & P-value \\
\hline Onset of pneumonia during hospitalization & 6.2 & $1.4 ; 9.9$ & 0.009 \\
\hline Eating difficulty & 2.3 & $0.6 ; 4.0$ & 0.007 \\
\hline
\end{tabular}

Table 3D: Mixed effect regression model comparing BOE data with systemic findings 


\begin{tabular}{|llll|}
\hline \multicolumn{4}{l}{ BOE - Total score } \\
\hline Variables & Coefficient $(\boldsymbol{\beta})^{1}$ & $95 \% \mathrm{Cl}$ & P-value \\
\hline 'Moderate' periodontitis at baseline & -1.0 & $-1.3 ;-0.5$ & $<0.001$ \\
\hline Regional ward (Reference: high-specialized ward) & -1.7 & $-2.8 ;-0.5$ & 0.006 \\
\hline 'Motor' domain over the study period & -0.6 & $-1.0 ;-0.1$ & 0.010 \\
\hline Dysphagia at baseline & 0.5 & $0.1 ; 1.1$ & 0.043 \\
\hline Age & 0.04 & $0.0 ; 0.1$ & 0.016 \\
\hline
\end{tabular}

\section{Supplementary Files}

This is a list of supplementary files associated with this preprint. Click to download.

- Appendix1SR20Nov20.doc

- Appendix1SR20Nov20.doc

- Appendix1SR20Nov20.doc

- Appendix1SR23Nov20.doc

- Appendix1SR23Nov20.doc

- Appendix1SR23Nov20.doc 